\title{
BMJ Open Danish Centre for Strategic Research in Type 2 Diabetes (DD2) project cohort of newly diagnosed patients with type 2 diabetes: a cohort profile
}

\author{
Diana Hedevang Christensen, ${ }^{1}$ Sia Kromann Nicolaisen, ${ }^{1}$ Klára Berencsi, ${ }^{1}$ \\ Henning Beck-Nielsen, ${ }^{2}$ Jørgen Rungby, ${ }^{3,4}$ Søren Friborg, ${ }^{5}$ Ivan Brandslund, ${ }^{6}$ \\ Jens Sandahl Christiansen, ${ }^{7}$ Allan Vaag, ${ }^{8}$ Henrik Toft Sørensen, ${ }^{1,9}$ \\ Jens Steen Nielsen, ${ }^{2}$ Reimar Wernich Thomsen ${ }^{1}$
}

To cite: Christensen DH, Nicolaisen SK, Berencsi K, et al. Danish Centre for Strategic Research in Type 2 Diabetes (DD2) project cohort of newly diagnosed patients with type 2 diabetes: a cohort profile. BMJ Open 2018;8:e017273. doi:10.1136/ bmjopen-2017-017273

- Prepublication history for this paper is available online. To view these files, please visit the journal online (http://dx.doi org/10.1136/bmjopen-2017017273).

Dr Jens Sandahl Christiansen died on 16 December 2015.

Received 20 April 2017 Revised 12 February 2018 Accepted 2 March 2018

Check for updates

For numbered affiliations see end of article.

Correspondence to Dr Reimar Wernich Thomsen; rwt@clin.au.dk

\section{ABSTRACT}

Purpose The aim of this article is to provide a detailed description of the ongoing nationwide Danish Centre for Strategic Research in Type 2 Diabetes (DD2) project cohort and biobank. The DD2 cohort continuously enrols newly diagnosed patients with type 2 diabetes (T2D) throughout Denmark. The overall goal of the DD2 project is to establish a large and data-rich T2D cohort that can serve as a platform for exhaustive T2D research including (1) improved genotypic and phenotypic characterisation of T2D, (2) intervention studies of more individualised T2D treatment, (3) pharmacoepidemiological studies and (4) long-term follow-up studies on predictors of T2D complications and prognosis.

Participants Between 2010 and 2016, 7011 individuals with T2D have been enrolled and assessed at baseline. Information collected include interview data (eg, body weight at age 20 years, physical activity and alcohol consumption), clinical examination data (eg, hip-waist ratio and resting heart rate) and biological samples (whole blood, DNA, plasma and urine) stored at $-80^{\circ} \mathrm{C}$ and currently analysed for a range of biomarkers and genotypes.

Findings to date Registry linkage has provided extensive supplemental continuous data on glycosylated haemoglobin A, lipids, albuminuria, blood pressure, smoking habits, body mass index, primary care contacts, hospital diagnoses and procedures, medication use, cancer and mortality. Cross-sectional associations between biomarkers, family history, anthropometric and lifestyle measures and presence of complications at baseline have been reported.

Future plans During 2016, a detailed follow-up questionnaire has been answered by $85 \%$ of initial participants, providing follow-up information on baseline variables and on presence of diabetic neuropathy. The DD2 cohort has now been followed for a total of 18862 person-years, and nested intervention trials and followup studies are ongoing. In the future, the cohort will serve as a strong national and international resource for recruiting patients to nested case studies, clinical trials, postmarketing surveillance, large-scale genome studies and follow-up studies of T2D complications.

\section{Strengths and limitations of this study}

- The Danish Centre for Strategic Research in Type 2 Diabetes (DD2) project cohort is an ongoing Danish cohort and biobank of newly diagnosed patients with type 2 diabetes (T2D) continuously enrolled on a nationwide level since 2010.

- By 2016, 7011 patients have been recruited from general practitioners $(53 \%)$ and hospital specialist outpatient clinics $(47 \%)$.

- The cohort includes baseline interview data, physical examination data, biological samples (DNA, blood and urine) as well as baseline and follow-up data on glycosylated haemoglobin A, lipids, albuminuria, blood pressure, smoking habits, body mass index, primary care contacts, hospital diagnoses and procedures, medication use, incident cancers, mortality and causes of death.

- The cohort may represent patients with more advanced T2D than average, and $85 \%$ already had initiated glucose-lowering treatment at enrolment that hampers studies of drug-naive patients with T2D.

- In the future, the DD2 cohort will serve as a strong national and international resource for recruiting patients to nested clinical trials, large-scale genome studies and long-term follow-up studies of $\mathrm{T} 2 \mathrm{D}$ complications through linkage with the large network of existing population-based registries in Denmark.

\section{INTRODUCTION}

Type 2 diabetes (T2D) is associated with a wide range of devastating complications, classically including cardiovascular disease, end-stage renal disease, blindness, diabetic foot ulcers and amputations. T2D also increases risk of infections, some cancers and neuropsychiatric disease. ${ }^{1}$ These complications inflict a burden on the healthcare system as well as on the individual patients with diabetes. Since it is hard to prevent T2D, there is a compelling 
need for cost-effective research to target and improve diabetes treatment in order to prevent complications. Fortunately, over the last decades, the number of available glucose-lowering treatment options has increased, ${ }^{2}$ facilitating individually tailored treatment. In this context determining how genotype and phenotype may influence the optimal choice of treatment is essential. ${ }^{3}$

Denmark has a tax-supported healthcare system that includes free access to care at general practitioners (GPs) and hospitals as well as partial reimbursement for most prescribed drugs, including diabetes drugs. ${ }^{4}$ GPs- the gatekeepers of the Danish National Health Service-are responsible for nearly all referrals to hospitals and specialists. Patient contacts with health and social services are extensively documented at the individual level in national databases using the unique civil personal registration number (CPR number) that has been assigned since 1968 to all Danish residents at birth or on immigration. ${ }^{4}$

In this setting, the nationwide Danish Centre for Strategic Research in Type 2 Diabetes (DD2) project was established in 2010. The Project's overall goal is to provide an international resource for T2D research. It encompasses establishment of the nationwide and data-rich DD2 cohort and a linked biobank of DNA, blood and urine. The study rationale has been to provide a platform for improved genotypic and clinical characterisation of T2D, for developing more individualised treatment and for testing new antidiabetic drugs in nested intervention studies. Moreover, to establish a unique T2D patient cohort by linkage with the large network of existing population-based registries in Denmark, through which patients can be followed longitudinally for a variety of clinical and socioeconomic outcomes for decades, allowing improved understanding of the predictors of long-term diabetes prognosis.

The aim of this cohort profile paper is to provide a description of the DD2 cohort as an international T2D research resource, including an overview of the collected data and a description of the baseline characteristics.

\section{COHORT DESCRIPTION}

\section{Study participants and recruitment}

Since 1 January 2009, all patients with newly or recently diagnosed T2D in Denmark have been eligible to participate in the DD2 cohort. The first patient was enrolled in November 2010, and the cohort now consists of 7011 patients. The DD2 project is ongoing, with continuous enrolment.

The process of enrolling in the DD2 cohort has been described in detail elsewhere ${ }^{5}$ : (1) clinical providers (usually either the patient's GP or a hospital physician/ nurse) identify patients newly diagnosed with T2D in routine clinical practice. (2) Those patients are informed by the clinical provider about the existence of the DD2 project. (3) Patients interested in participating receive detailed oral and written information and are asked to sign a written informed consent document for enrolment. (4) Patient clinical information is then collected: GPs or hospital physicians/nurses complete an online questionnaire, including items requiring a physical examination. (5) Urine and fasting blood samples are collected for storage in a biobank. ${ }^{6}$

Approximately $80 \%$ of patients with T2D in Denmark receive care at GPs' offices and the remainder at hospital specialist outpatient clinics. Enrolment into the DD2 cohort takes place in both settings (figure 1). From 2010 to 2012, most patients were enrolled at hospital outpatient clinics rather than at GP offices (1559 vs 739 patients). From 2013 on, the number of patients recruited by GPs increased rapidly, ${ }^{7}$ reaching 3688 in February 2016. As of that month, the corresponding number of patients recruited by outpatient specialist clinics was 3323 (table 1).

During the entire DD2 enrolment period, all diagnosing of T2D in routine clinical practice has been made according to WHO criteria, before 2012 primarily based on OGTT and after 2012 primarily based on glycosylated haemoglobin A (HbAlc) $>48 \mathrm{mmol} / \mathrm{mol}(6.5 \%)$. No further diagnostic criteria for enrolment have been applied in the DD2 project. The DD2 project explicitly aims to comprehensively study T2D as diagnosed in everyday clinical care, as one of the project aims is to document pitfalls in initial T2D diagnosing, including investigation of subtypes and subphenotypes in the cohort, occurrence of secondary diabetes, autoimmune diabetes and so on.

While the DD2 cohort from the beginning aimed to focus on newly diagnosed T2D patients, in clinical practice, the referral to DD2 may not happen at first diabetes notice when other clinical activity may be more pertinent. Individuals who have had prevalent T2D for some time after 2009 are also accepted for participation. While we do not have complete data on exact date of diabetes diagnosis for all individuals, average time from first recorded glucose-lowering drug initiation to enrolment date in the DD2 cohort is $1-1.5$ years.

The exact proportion of all patients with incident T2D in Denmark that is enrolled into the DD2 cohort is unknown. With an average enrolment in the order of 1000-1200 DD2 patients per year, the project enrols an estimated $5 \%$ of newly diagnosed patients with T2D nationwide.

The number of enrolled patients and recruitment sites vary across the five Danish healthcare regions. Currently, the largest proportion $(35 \%)$ of the cohort patients has been recruited from the region of Southern Denmark, which comprises $21 \%$ of Denmark's population, followed by the Central Denmark Region (with 24\% of cohort patients) and the Capital Region (with 19\% of cohort patients), comprising $22 \%$ and $31 \%$ of Denmark's population, respectively.

Loss of patients from the DD2 cohort can occur due to emigration and death. These events are identified by linkage to the Danish Civil Registration System (CRS), which has recorded vital status for the entire Danish population since 1968, with daily electronic updates. ${ }^{4}$ Enrolled 


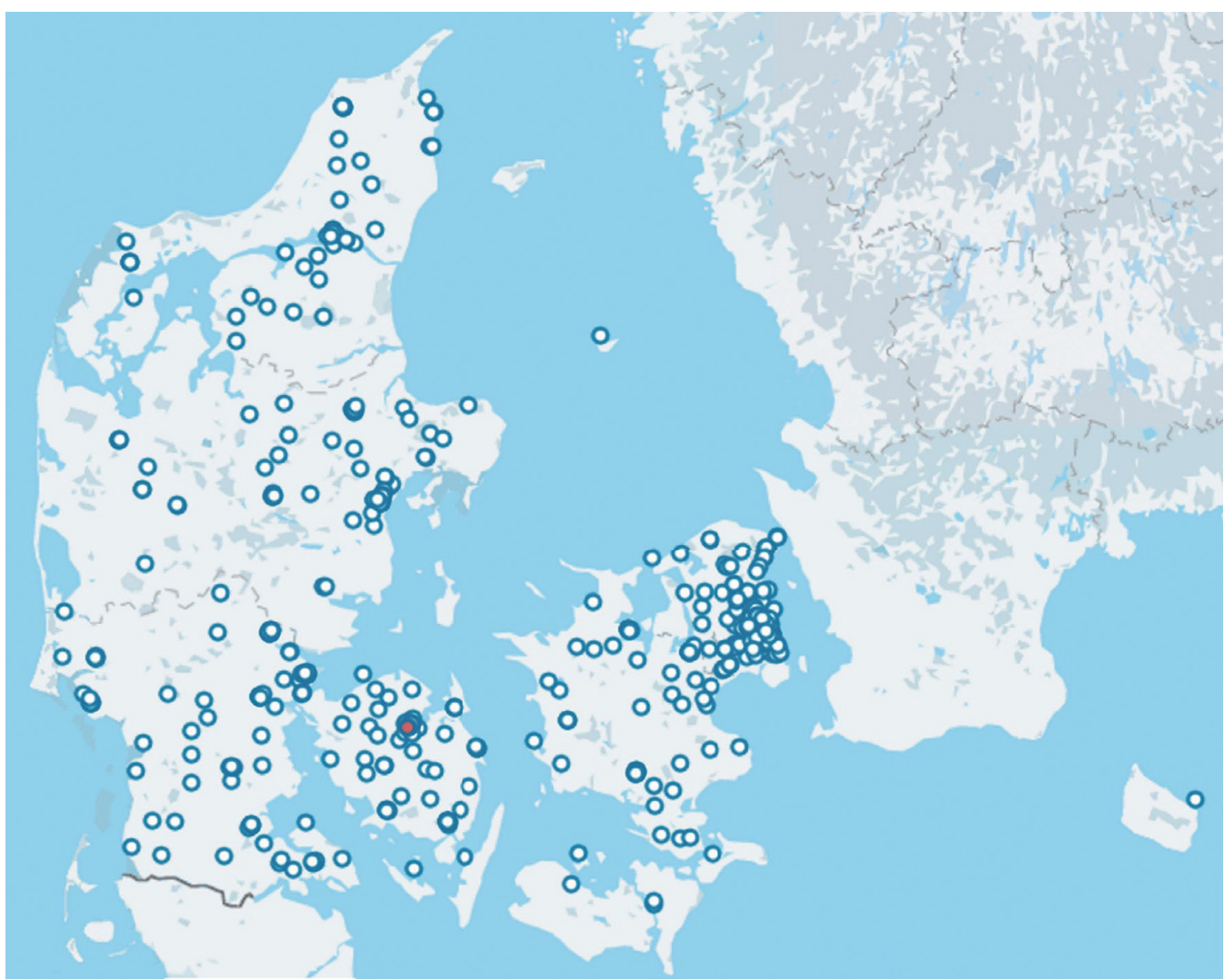

Figure 1 Enrolment sites throughout Denmark. Every circle represents an enrolment site, either at a general practitioner's office or at an outpatient clinic. The administrative headquarter of the DD2 is in Odense (red dot). DD2, Danish Centre for Strategic Research in Type 2 Diabetes.

patients have the right to withdraw from the DD2 cohort, but only four individuals have done so thus far.

\section{Data collection}

Baseline data recorded in the DD2 database include each patient's CPR number, date of enrolment and DD2 variables collected directly at the enrolment visit (interview data, clinical examination data and biobank data). Furthermore, linkage with a wide range of Danish health and administrative registries, including the Danish Diabetes Database for Adults (DDDA), one of several Danish nationwide public clinical quality improvement registries ${ }^{7}$ ensures important additional baseline data (figure 2).

The cohort is followed prospectively through linkage with the DDDA and other Danish administrative and health registries. Moreover, directly collected data are used to follow DD2 patients for outcomes not routinely available in medical registries as described below.

Directly collected DD2 baseline variables

These include waist-hip ratio, recalled body weight at age 20 years, maximum lifetime body weight, alcohol consumption, family history of diabetes, resting heart

Table 1 Geographical distribution of DD2 participants by recruitment setting and year of enrolment

\begin{tabular}{|c|c|c|c|c|c|c|c|c|c|c|c|c|c|c|c|}
\hline \multirow[b]{2}{*}{ Region } & \multicolumn{8}{|c|}{ Hospital outpatient clinics, N } & \multicolumn{7}{|c|}{ General practitioners' offices, $\mathbf{N}$} \\
\hline & 2010 & 2011 & 2012 & 2013 & 2014 & 2015 & 2016 & Total & 2011 & 2012 & 2013 & 2014 & 2015 & 2016 & Total \\
\hline Capital & 0 & 23 & 246 & 197 & 199 & 85 & 5 & 755 & 7 & 144 & 259 & 134 & 39 & 5 & 588 \\
\hline North Denmark & 0 & 2 & 52 & 16 & 73 & 19 & 5 & 167 & 0 & 31 & 276 & 161 & 30 & 0 & 498 \\
\hline Zealand & 0 & 0 & 0 & 6 & 20 & 4 & 0 & 30 & 3 & 1 & 432 & 239 & 71 & 11 & 757 \\
\hline Unspecified & - & - & - & - & - & - & - & - & - & 2 & 29 & 7 & 1 & 2 & 41 \\
\hline Total Denmark & 18 & 570 & 957 & 686 & 674 & 396 & 22 & 3323 & 194 & 540 & 1425 & 772 & 684 & 73 & 3688 \\
\hline
\end{tabular}

DD2, Danish Centre for Strategic Research in Type 2 Diabetes. 


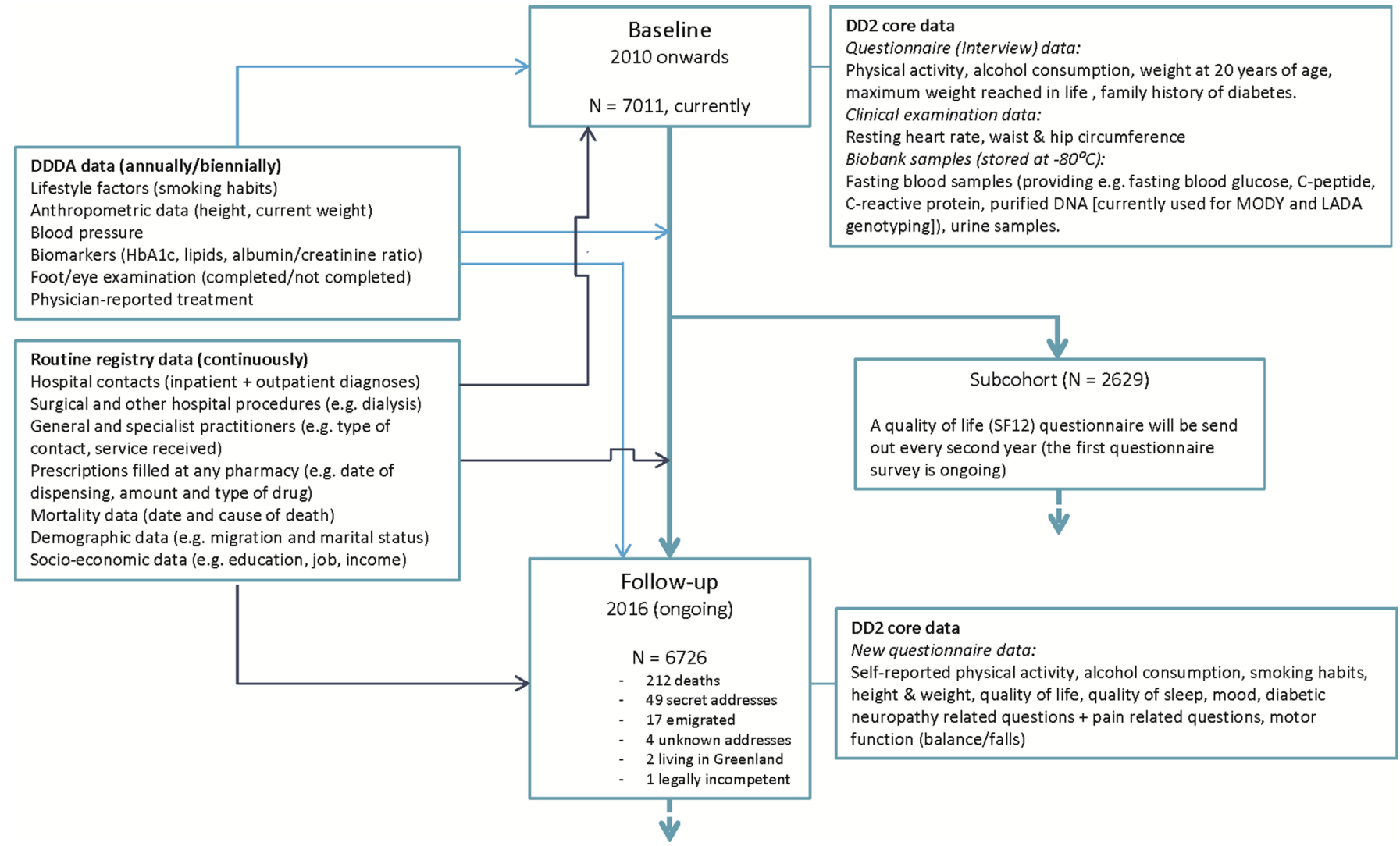

Figure 2 Flow chart of data collection in the DD2 cohort study. DD2, Danish Centre for Strategic Research in Type 2 Diabetes; DDDA, Danish Diabetes Database for Adults; HbA1c, glycosylated haemoglobin A; SF-12, 12-Item Short Form Health Survey.

rate, physical activity level and (added in 2015) self-reported date of first T2D diagnosis. The self-reported physical activity level is currently being validated by placing accelerometers (AX3, Axivity, Newcastle, UK) directly on the skin of the thigh and back of persons in a subcohort of DD2 participants $(\mathrm{n}=1000)$, and all physical activity is recorded over a 10-day period. Moreover, a smartphone application (www.interwalk.dk) holding an interval-based training program has been developed. DD2 participants are encouraged to download and use this application, which guides and monitors their physical exercise. The logged exercise data are then transferred to the DD2 cohort and allow future investigations of for example, the impact of exercise on risk of T2D complications. Urine and blood samples (whole blood, serum, plasma and purified DNA) are stored in a biobank ${ }^{6}$ and can be used for myriad purposes. To date, plasma samples have been analysed for a number of baseline variables, including fasting blood glucose, C-peptide (used in homeostatic model assessment of insulin resistance), glutamic acid decarboxylase antibodies, alanine-aminotransferases (ALAT), amylase and C reactive protein (CRP). Several currently initiated and planned studies will provide a larger range of analysed biobank variables. For instance, DNA has been purified from 6000 patients and 'maturity-onset diabetes of the young' (MODY) genes are now being sequenced in order to identify individuals carrying likely pathogenic variants. As well, studies of the prevalence of 'latent autoimmune diabetes in adults' in cohort members and the impact of the inflammatory system on diabetic complications have been initiated and will lead to analyses of additional biomarkers, including mannan-binding lectin.

\section{Variables provided by the DDDA}

The DDDA, established in 2005, provides a key source of supplemental individual-level data. ${ }^{7}$ Quality of care data (variables outlined below) are submitted from GP offices and outpatient clinics to the DDDA annually or biennially. The DDDA thus can provide baseline and follow-up data for members of the DD2 cohort including data on the T2D diagnosis date first recorded in the healthcare system, tobacco smoking, completion of examinations for diabetic foot and eye disease, body mass index (BMI), physician-reported antidiabetic treatment, antihypertensive treatment, hypolipidaemic treatment and routine laboratory measurements such as HbAlc, plasma lipids and albuminuria.

\section{Administrative and health registries}

Migration and civil status can be ascertained from the CRS $^{4}$ (figure 3). The Danish National Patient Registry $(\mathrm{DNPR})^{8}$ maintains complete data on all hospital admissions since 1977 and on all hospital outpatient clinic and emergency room contacts since 1995, including dates of admission and discharge, visit dates, surgical procedures 


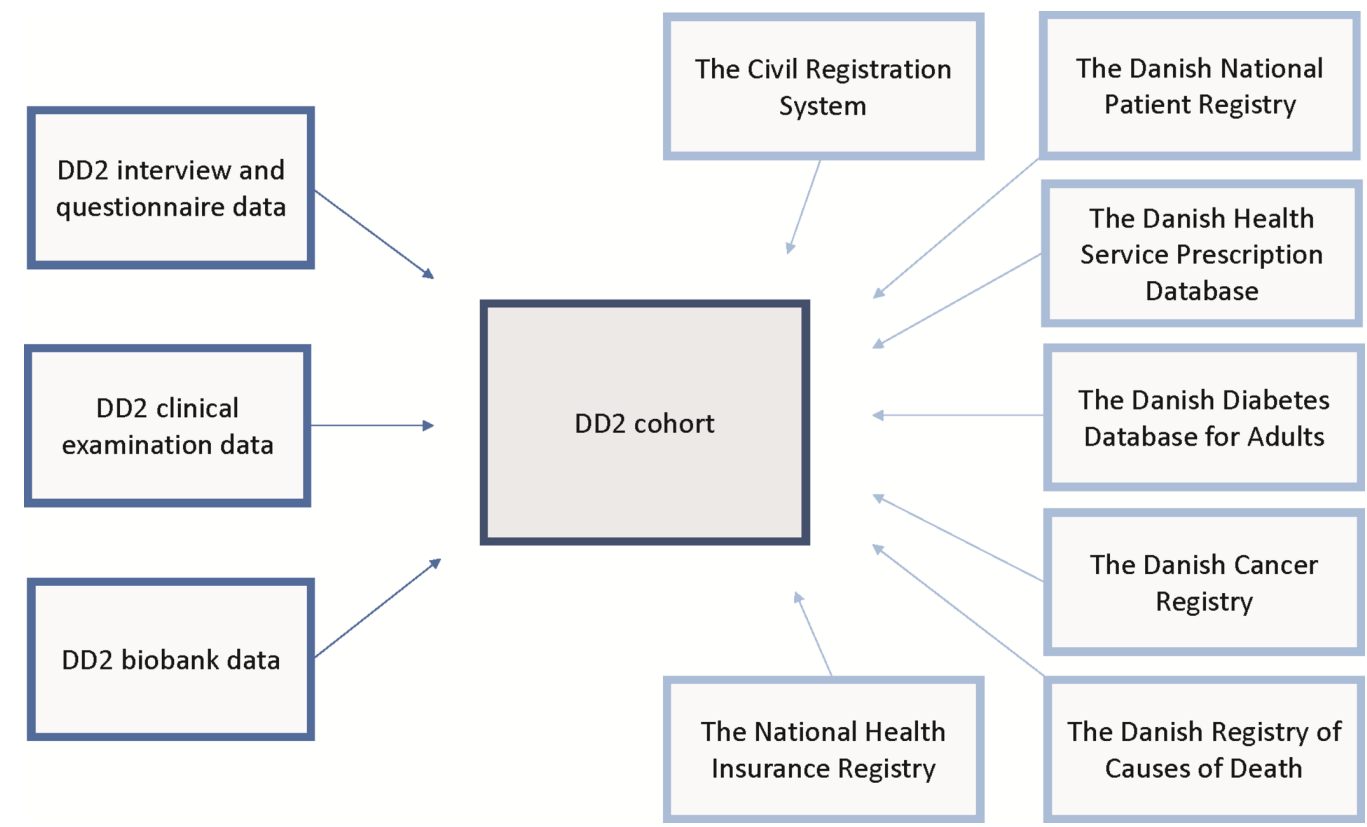

Figure 3 Schematic overview of individual-level data linkage in the DD2 cohort using the civil registration number as personal identification. DD2, Danish Centre for Strategic Research in Type 2 Diabetes.

performed and primary and secondary discharge diagnoses coded according to the International Classification of Diseases, 8th Revision until the end of 1993 and 10th Revision thereafter. Linkage to the DNPR makes it possible to obtain individual-level information at baseline and during follow-up on diseases and treatments relevant to diabetes, such as cardiovascular events, microvascular complications and dialysis. On the basis of diagnoses in the DNPR, the baseline comorbidity burden of each DD2 participant is calculated using Charlson Comorbidity Index (CCI) $\operatorname{scores}^{9}$ (low: 0 points, medium: 1-2 points, high $\geq 3$ points; diabetes is excluded as it constitutes the index disease). Linkage to the Danish National Health Service Prescription Database (DNHSP ${ }^{10}$ provides individual-level information on reimbursable drugs dispensed at all pharmacies in Denmark allowing to track treatment history of each DD2 participant. Pharmacy data include the date of dispensing, as well as amount and type of drug prescribed according to the Anatomical Therapeutic Chemical classification system. The Danish Cancer Registry (DCR) ${ }^{11}$ has recorded data on cancer diagnoses in Denmark since 1943 and is a valuable resource for studying cancer in patients with T2D. Linkage to the Danish Register of Causes of Death (DRCD) ${ }^{12}$ allows evaluation of cause-specific mortality, while linkage to the Statistics Denmark database provides socioeconomic data, for example, on education, job level and income. The National Health Insurance Service Registry contains information on all services provided by GPs and specialists in Denmark since 1990, including physiotherapists, dentists and chiropractors. Data on services include the number of contacts, health service provider and type of service (eg, consultation, blood glucose measurement or vaccination). ${ }^{13}$

\section{Questionnaire data 2016}

By June 2016, all enrolled DD2 patients were sent questionnaires to gather follow-up data (described below) as well as data on the prevalence of diabetic neuropathy. Neuropathy-related questions included, among others, the Michigan Neuropathy Screening Instrument questionnaire, the Douleur Neuropathique 4 questionnaire and other pain-related questions. A random sample of patients reporting symptoms of painful and non-painful neuropathy as well as a random sample of control patients without symptoms will be invited for an in-depth neuropathy examination during 2016-2018, which will allow comprehensive investigation of this late diabetic complication. The questionnaires also maintained follow-up questions regarding the lifestyle factors included in the baseline study interview, including alcohol use, smoking habits and physical activity (figure 2). Preliminary data from this questionnaire survey have revealed a remarkable response rate of $85 \%$.

Also, quality of life 12-Item Short Form Health Survey (SF-12) questionnaires have recently been emailed to all DD2 cohort members with a known email address $(n=2629)$. As of August 2016, 1002 patients (38\% of those with email addresses) had responded to this questionnaire. Follow-up SF-12 questionnaires will be emailed automatically every second year.

\section{Baseline characteristics of study participants}

An overview of the interview and clinical examination data collected from the 7011 patients enrolled in the DD2 between November 2010 and February 2016 is provided in table 2.

As of February 2016, the DD2 cohort consisted of $4065(58 \%)$ men, the median age was 61 years (IQR: 
Table 2 Characteristics of patients with newly diagnosed type 2 diabetes enrolled in the Danish Centre for Strategic Research in Type 2 Diabetes (DD2) project during November 2010-February 2016

\begin{tabular}{|c|c|}
\hline Variables & $\begin{array}{l}\text { Patients with } \\
\text { type } 2 \text { diabetes }\end{array}$ \\
\hline $\begin{array}{l}\text { Persons enrolled in DD2 by February, } \\
2016, n(\%)\end{array}$ & $7011(100)$ \\
\hline $\begin{array}{l}\text { Enrolled from general practitioners' } \\
\text { offices, } n(\%)\end{array}$ & $3688(53)$ \\
\hline Enrolled from hospital clinics, n (\%) & $3323(47)$ \\
\hline Median age (IQR), years & $61(52-68)$ \\
\hline Minimum-maximum age, years & $17-95$ \\
\hline \multicolumn{2}{|l|}{ Gender, n (\%) } \\
\hline Male & $4065(58)$ \\
\hline Female & $2946(42)$ \\
\hline Resting heart rate, median (IQR) & $70(63-79)$ \\
\hline $\begin{array}{l}\text { Waist circumference, median } \\
(\mathrm{IQR}), \mathrm{cm}\end{array}$ & $106(97-116)$ \\
\hline Waist-hip ratio in men, median (IQR) & $1.02(0.97-1.06)$ \\
\hline Waist-hip ratio in women, median (IQR) & $0.92(0.87-0.97)$ \\
\hline Median weight at 20 years of age (IQR), $\mathrm{kg}$ & $70(60-80)$ \\
\hline Weight unknown at age 20, n (\%) & $929(13)$ \\
\hline Median maximum lifetime weight (IQR), $\mathrm{kg}$ & $100(86-115)$ \\
\hline $\begin{array}{l}\text { Maximum lifetime weight unknown, n } \\
(\%)\end{array}$ & $75(1)$ \\
\hline \multicolumn{2}{|l|}{ Alcohol use, n (\%) } \\
\hline $\begin{array}{l}\text { Maximum 14/21 drinks/week (women/ } \\
\text { men) }\end{array}$ & $6539(93)$ \\
\hline $\begin{array}{l}\text { More than } 14 / 21 \text { drinks/week (women/ } \\
\text { men) }\end{array}$ & $472(7)$ \\
\hline
\end{tabular}

Days per week with $30+$ min of physical activity (days)

\begin{tabular}{|ll}
0 & $1161(17)$ \\
1 & $488(7)$ \\
2 & $891(13)$ \\
3 & $954(14)$ \\
4 & $622(9)$ \\
5 & $681(10)$ \\
6 & $317(5)$ \\
7 & $1897(27)$ \\
Regular sports activities, $n(\%)$ & $2748(39)$ \\
\hline Yes & $4262(61)$ \\
\hline No & \\
\hline
\end{tabular}

Level of physical activity during the past

year, $\mathrm{n}(\%)$

Hard physical training and competitive 55 (1)

sports several times a week

Leisure sports, heavy garden work or 1370 (20)

similar activity at least 4 hours per week
Table 2 Continued

\section{Variables}

Patients with type 2 diabetes

Walking, cycling or other light exercise 4298 (61) at least 4 hours per week

Reading, television watching or other 1288 (18) sedentary activity

Family history of diabetes, n (\%)

Yes 3717 (53)

No 2696 (39)

Don't know 598 (9)

$\begin{array}{ll}\text { Median fasting blood glucose (IQR), } & 7.1(6.4-8.3) \\ \mathrm{mmol} / \mathrm{L}^{*} & \\ \text { Median C-peptide†‡ (IQR), pmol/L } & \begin{array}{l}1161(865- \\ 1570)\end{array}\end{array}$

Glutamic acid decarboxylase antibodies§ $\leq 30 \mathrm{kU} / \mathrm{L}, \mathrm{N}(\%)$ $5655(97)$

$>30 \mathrm{kU} / \mathrm{L}, \mathrm{N}(\%)$ 161 (3)

Median alanine-aminotransferases (IQR), 27 (20-38) U/L

Median pancreatic amylaseף (IQR), U/L 23 (16-30) Median C reactive proteinף (IQR), mg/L 2.1 (1.0-4.8)

${ }^{*}$ Currently analysed for the first consecutive 5363 DD2 patients. †Reference range: $400-1600 \mathrm{pmol} / \mathrm{L}$.

‡Currently analysed for the first consecutive 5800 DD2 patients. §Currently analysed for the first consecutive 5816 DD2 patients. ๆCurrently analysed for the first consecutive 1018 DD2 patients.

52-68 years) and $3717(53 \%)$ patients had a known family history of diabetes. In regard to exercise, 1161 patients (17\%) reported less than $30 \mathrm{~min}$ and 1897 patients $(27 \%)$ reported at least $30 \mathrm{~min}$ of physical activity 7 days per week. Overall, 2748 patients (39\%) reported regular sports activities. At baseline, 7\% $(n=472)$ consumed more alcohol per week than the recommended maximum safe amount in Denmark (maximum of 14/21 drinks per week for women/ men). Weight at 20 years of age was available for 6082 study participants, with a median value of $70 \mathrm{~kg}$ (IQR: $60-80 \mathrm{~kg})$. The median value of maximum lifetime weight was $100 \mathrm{~kg}$ (IQR: $86-115 \mathrm{~kg}$ ) in the cohort. Median waist-hip ratios were 1.02 (IQR: 0.97-1.06) in men and 0.92 (IQR: $0.87-0.97$ ) in women. To date, biological samples have been obtained from more than $93 \%$ of cohort members, with a median fasting blood glucose measurement of $7.1 \mathrm{mmol} / \mathrm{L}$ (minimum-maximum: $2.5-29 \mathrm{mmol} / \mathrm{L}$ ) and a median C-peptide measurement of $1161 \mathrm{pmol} / \mathrm{L}$ (IQR: $865-1570 \mathrm{pmol} / \mathrm{L}){ }^{14}$

By September 2015, 5115 patients $(73 \%)$ had been linked to the DDDA quality-of-care database (table 3).

Of the 3835 patients with a known BMI value, $37 \%$ of men were overweight at DD2 enrolment and 52\% were obese, while $29 \%$ of women were overweight and $55 \%$ were obese. Seventeen per cent $(n=886)$ were 
Table 3 Characteristics of 5115 patients enrolled in the nationwide Danish Centre for Strategic Research in Type 2 Diabetes (DD2) project, which currently can be linked to the Danish Diabetes Database for Adults

Variables

N (\%) of DD2 participants who could be $5115(73)$
linked to the Danish Diabetes Database
for Adults, as of September 2015.

Tobacco smoking, $\mathrm{n}$ (\%)

\begin{tabular}{|ll}
\hline Never smoker & $2254(44)$ \\
\hline Former smoker & $1649(32)$ \\
\hline Current smoker, daily & $886(17)$ \\
\hline Current smoker, occasionally & $55(1)$ \\
\hline Smoking status listed as unknown & $271(5)$ \\
\hline Men (n=2984) & \\
\hline Median height (IQR), cm & $178(174-183)$ \\
\hline Height missing, $\mathrm{n}(\%)$ & $709(24)$ \\
\hline Current median weight (IQR), kg & $96(85-110)$ \\
\hline Weight missing, n (\%) & $229(8)$ \\
\hline Current BMI, n (\% of those with known BMI) & \\
\hline$<18.5$ & $2(0)$ \\
\hline 18.5-24.9 & $250(11)$ \\
\hline 25-29.9 & $833(37)$ \\
\hline 30+ & $1183(52)$ \\
\hline BMI missing & 716 \\
\hline Women (n=2131) & \\
\hline Median height (IQR), cm & $164(160-169)$ \\
\hline Height missing, $\mathrm{n}(\%)$ & $561(26)$ \\
\hline Current median weight (IQR), kg & $188(4)$ \\
\hline Weight missing, $\mathrm{n}(\%)$ & \\
\hline Current BMI, n (\% of those with
\end{tabular}

Current BMI, n (\% of those with known BMI)

\begin{tabular}{|ll}
\hline$<18.5$ & $8(1)$ \\
\hline $18.5-24.9$ & $240(15)$ \\
$25-29.9$ & $458(29)$ \\
$30+$ & $861(55)$ \\
\hline BMI missing & 564 \\
\hline HbA1c value & \\
\hline$<7 \%$ & $3592(70)$ \\
\hline $7.0 \%-8.0 \%$ & $824(16)$ \\
\hline $8.0 \%-9.0 \%$ & $303(6)$ \\
\hline$\geq 9.0 \%$ & $313(6)$ \\
\hline HbA1c missing & $83(2)$ \\
\hline Albuminuria, N (\%) & \\
\hline Albumin-creatinine ratio $<30 \mathrm{mg} / \mathrm{g}$ & $3565(70)$ \\
\hline Albumin-creatinine ratio $30-300 \mathrm{mg} / \mathrm{g}$ & $830(16)$ \\
\hline Albumin-creatinine ratio $\geq 300 \mathrm{mg} / \mathrm{g}$ & $110(2)$ \\
\hline
\end{tabular}

Continued
Table 3 Continued

Patients with type 2

Variables diabetes

Albuminuria-creatinine ratio missing $610(12)$

Blood pressure, median (IQR), $\mathrm{mm} \mathrm{Hg}$

Systolic

$130(124-140)$

Diastolic $80(74-85)$

Lipids, median (IQR), mmol/L

Total cholesterol $4.4(3.7-5.1)$

HDL cholesterol $1.2(1.0-1.5)$

LDL cholesterol $2.2(1.7-2.8)$

Triglycerides $1.6(1.2-2.4)$

Physician-reported antidiabetic treatment, $\mathrm{n}$ $(\%)^{*}$

$\begin{array}{ll}\text { Insulin only } & 59(1) \\ \text { Insulin and oral antidiabetic treatment } & 306(6) \\ \text { Oral antidiabetic treatment } & 3993(78) \\ \text { None } & 757(15) \\ \text { Physician-reported antihypertensive } & 3523(69) \\ \text { reatment, n (\%) } & \end{array}$

Physician-reported ACE inhibitor or ATII- 2786 (54)
antagonist treatment, $n(\%)$

Physician-reported hypolipidaemic treatment 3373 (66)

$\begin{array}{ll}\text { Eye screening completed } & 2762(54) \\ \text { Foot examination completed } & 4362(85)\end{array}$

*Within the year prior to enrolment in the DD2 cohort. ATII-antagonist, angiotensin 2 antagonist; BMI, body mass index; HbA1c, glycosylated haemoglobin A; HDL, high-density lipoprotein; LDL, low-density lipoprotein.

current daily smokers, and $32 \%(\mathrm{n}=1649)$ were former smokers. Most had an HbA1c value below 7.0\% (70\%) and $4358(85 \%)$ had received antidiabetic treatment within the year prior to enrolment; 59 patients $(1 \%)$ were taking only insulin and $3993(78 \%)$ received only oral antidiabetic treatment, as confirmed by routine prescription data from DNHSP. A diabetic eye examination had been performed on 2762 (54\%) cohort members and a foot examination on $4362(85 \%)$. At DD2 enrolment, microalbuminuria was present in $16 \%$ of patients, and macroalbuminuria was present in $2 \%$. Median systolic/diastolic blood pressure was 130/80, and 3523 patients $(69 \%)$ were being treated for hypertension. Median total cholesterol was 4.4 (IQR: $3.7-5.1) \mathrm{mmol} / \mathrm{L} ; 3373$ patients $(66 \%)$ were receiving hypolipidaemic treatment. Linkage of the DD2 cohort to the DNPR and DNHSP revealed that approximately one-third of patients had hospital-diagnosed comorbidity (CCI score $\geq 1$ point) prior to enrolment. Twenty-two per cent had hospital-diagnosed macrovascular disease, and $15 \%$ had microvascular complications prior to enrolment (table 4). 
Table 4 Comorbidities (determined through linkage with the DNPR) and use of medications (determined through linkage with the DNHSP) at enrolment among the first 7011* patients with newly diagnosed type 2 diabetes enrolled in the DD2 project

\begin{tabular}{|c|c|}
\hline Variables & $\begin{array}{l}\text { Patients with type } \\
2 \text { diabetes }\end{array}$ \\
\hline $\begin{array}{l}\text { Persons enrolled in DD2 by February } \\
2016, n(\%)\end{array}$ & $7011(100)$ \\
\hline \multicolumn{2}{|l|}{ Charlson Comorbidity Index score† } \\
\hline 0 & $4807(69)$ \\
\hline $1-2$ & $1820(26)$ \\
\hline $3+$ & $384(5)$ \\
\hline
\end{tabular}

Charlson Comorbidity Index conditions at

baseline, $\mathrm{n}(\%)$

\begin{tabular}{|c|c|}
\hline Myocardial infarction & $345(5)$ \\
\hline Congestive heart failure & $283(4)$ \\
\hline Peripheral vascular disease & $309(4)$ \\
\hline Cerebrovascular disease & $465(7)$ \\
\hline Dementia & $13(0.2)$ \\
\hline Chronic pulmonary disease & $510(7)$ \\
\hline Connective tissue disease & $183(3)$ \\
\hline Ulcer disease & $126(2)$ \\
\hline Mild liver disease & $111(2)$ \\
\hline Hemiplegia & $19(0.3)$ \\
\hline Moderate to severe renal disease & $117(2)$ \\
\hline Any tumour & $508(7)$ \\
\hline Leukaemia & $15(0.2)$ \\
\hline Lymphoma & $26(0.4)$ \\
\hline Moderate to severe liver disease & $16(0.2)$ \\
\hline Metastatic solid tumour & $43(0.6)$ \\
\hline HIV/AIDS & $9(0.1)$ \\
\hline Any macrovascular complications & $1511(22)$ \\
\hline Any microvascular complications & $1035(15)$ \\
\hline Diabetic neuropathy & $230(3)$ \\
\hline Diabetic retinopathy & $682(10)$ \\
\hline Diabetic nephropathy & $209(3)$ \\
\hline $\begin{array}{l}\text { Mental disorder (based on discharge and } \\
\text { treatment codes for mental illness) }{ }^{*}\end{array}$ & $1456(21)$ \\
\hline \multicolumn{2}{|l|}{ Antidiabetic treatment, $\mathrm{n}(\%)^{\star} \ddagger$} \\
\hline Insulin only & $80(1)$ \\
\hline Insulin and oral antidiabetic treatment & $396(6)$ \\
\hline Oral antidiabetic treatment & $5387(78)$ \\
\hline None & $1053(15)$ \\
\hline \multicolumn{2}{|l|}{ Glucose-lowering drugs, $n(\%)^{\star} \ddagger$} \\
\hline Biguanides (metformin) & $5631(81)$ \\
\hline Insulin & $476(7)$ \\
\hline Sulfonylureas & $463(7)$ \\
\hline DPP-4 inhibitors & $653(9)$ \\
\hline GLP-1 analogues & $378(5)$ \\
\hline SGLT-2 inhibitors & $57(0.8)$ \\
\hline
\end{tabular}

Continued
Table 4 Continued

\begin{tabular}{lc} 
Variables & $\begin{array}{l}\text { Patients with type } \\
\text { 2 diabetes }\end{array}$ \\
\hline Meglitinides & $7(0.1)$ \\
\hline Thiazolidinediones (glitazones) & $1(0.01)$ \\
\multicolumn{1}{|c|}{ Alpha-glucosidase inhibitors } & $4(0.06)$ \\
$\begin{array}{l}\text { Antihypertensive treatment excluding } \\
\text { loopdiuretics, } n(\%)^{\star} \ddagger\end{array}$ & $4991(72)$
\end{tabular}

ACE inhibitors or ATII-antagonist treatment, 4157 (60) $\mathrm{n}(\%)^{\star} \ddagger$

$\begin{array}{lc}\text { Hypolipidaemic treatment, } \mathrm{n}(\%)^{*} \ddagger & 4886(71) \\ \text { Oral steroids, } \mathrm{n}(\%)^{*} \ddagger & 430(6)\end{array}$

*At the time of analysis, full prescription data were available only until 31 December 2015. Therefore, variables based on data from the DNHSP are for a subcohort numbering 6916 patients. †Diabetes mellitus not included in the score as it constitutes the index disease. Comorbidity categories include DNPR derived data on cancer; more detailed cancer data are available from the Danish Cancer Registry.

¥Within the last year prior to DD2 enrolment.

ATII-antagonist, angiotensin 2 antagonist; DD2, Danish Centre for Strategic Research in Type 2 Diabetes; DNPR, Danish National Patient Registry; DNHSP, Danish National Health Service Prescription; DPP-4-inhibitors, dipeptidylpeptidase 4 inhibitors; GLP-1 analogues, glucagon-like peptide 1 analogues; SGLT-2 inhibitors, sodium glucose cotransporter 2 inhibitors.

\section{FINDINGS TO DATE \\ Mortality ratios}

The DD2 cohort has been followed for a total of 18862 person-years (PY) and a mean of 2.7 years. A total of 212 patients have died, yielding an all-cause mortality rate (MR) of 1.12 per 100 PY (95\% CI 0.97 to 1.29), with differences by gender (MR for women: 0.81 (95\% CI 0.62 to 1.03 ); MR for men: 1.36 (95\% CI 1.15 to 1.60$)$ ). As expected, all-cause MR increased with age (table 5).

Table 5 Number of deaths and overall, age-specific and gender-specific mortality rates (per 100 person-years) for the baseline cohort of 7011 DD2 patients

\begin{tabular}{|lll|}
\hline Variables & $\begin{array}{l}\text { Number of } \\
\text { deaths }\end{array}$ & MR (95\% Cl) \\
\hline $\begin{array}{l}\text { Total DD2 cohort } \\
\text { Gender }\end{array}$ & 212 & $1.12(0.97$ to 1.29$)$ \\
\hline $\begin{array}{l}\text { Women } \\
\text { Men }\end{array}$ & 65 & $0.81(0.62$ to 1.03$)$ \\
\hline $\begin{array}{c}\text { Age (years) } \\
<40\end{array}$ & 147 & $1.36(1.15$ to 1.60$)$ \\
\hline $40-59$ & 37 & $0.23(0.03$ to 0.83$)$ \\
$60-79$ & 149 & $0.50(0.35$ to 0.69$)$ \\
$\geq 80$ & 24 & $1.47(1.25$ to 1.73$)$ \\
\hline
\end{tabular}

Median follow-up time from date of enrolment to death or to date of latest data extraction from the Civil Registration System was 2.68 years (IQR: $1.67-3.63$ years).

DD2, Danish Centre for Strategic Research in Type 2 Diabetes; MR, mortality rate. 


\section{Key findings}

Several baseline cross-sectional studies based on preliminary findings for parts of the current DD2 cohort were published in 2014-2016. ${ }^{15-20}$ Parental history of T2D was associated with younger age at diagnosis (adjusted prevalence ratio (aPR) for age <40 years: 1.66 (95\% CI 1.19 to 2.31); aPR for age 40-60 years: 1.36 (95\% CI 1.24 to $1.48)$ ), as well as poorer glucose control at diagnosis (aPR for fasting blood glucose <6.5 mmol/L: 0.80 (95\% CI 0.62 to 1.02 ) and aPR for fasting blood glucose $\geq 7.5 \mathrm{mmol} / \mathrm{L}$ : 1.47 (95\% CI 1.20 to 1.80)). No associations were observed for anthropometric and lifestyle factors, while parental history tended to be negatively associated with the high beta cell function phenotype ${ }^{18}$ (aPR 0.79 (95\% CI 0.61 to 1.01)). This suggests that patients with T2D may not inherit diabetes primarily as a result of family-learned lifestyle habits (physical inactivity and overeating), but rather as a result of a constitutional impairment of insulin action/secretion. ${ }^{18}$ In studies of DD2 biobank data, a $16 \%$ prevalence of elevated ALAT ( $>38 \mathrm{IU} / \mathrm{L}$ for women and $>50 \mathrm{IU} / \mathrm{L}$ for men $)^{15}$ and a $40 \%$ prevalence of elevated CRP $(>3.0 \mathrm{mg} / \mathrm{L})^{16}$ have been found among newly diagnosed patients with T2D. As well, potentially modifiable predictors of elevated ALAT/CRP have been identified, including low physical activity (ALAT: relative risk (RR) $1.4,95 \%$ CI 1.04 to 1.93; CRP: RR $1.46,95 \%$ CI 1.11 to 1.91$).{ }^{1516}$ Such biomarkers may have an impact on the clinical outcome of diabetes, which future prospective studies using DD2 data will be able to examine.

In another study, patients with incident T2D were followed during their first year postdiagnosis. It was found that $74 \%$ were receiving glucose-lowering therapy, with $88 \%$ receiving monotherapy, mainly metformin. Factors associated with receiving any glucose-lowering therapy included young age (RR 1.29 , 95\% CI 1.16 to 1.44 ), central obesity ( $\mathrm{RR}=1.23,95 \% \mathrm{CI} 1.04$ to 1.44$)$, large weight gain since youth ( $R R=1.10,95 \%$ CI 1.03 to 1.18$)$, lack of regular physical activity $(\mathrm{RR}=1.07,95 \% \mathrm{CI} 1.01$ to 1.15), high baseline fasting blood glucose $(\mathrm{RR}=1.25$, $95 \% \mathrm{CI} 1.10$ to 1.42 ) and high comorbidity burden $(\mathrm{RR}=1.20,95 \%$ CI 1.05 to 1.38$) .{ }^{17}$

A publication list is provided at www.dd2.nu. The results of many ongoing long-term prospective studies will be reported in years to come including genotype studies investigating the association between genotypes and pharmacological treatment strategies on relevant endpoints like glycaemic control and albuminuria.

\section{STRENGTHS AND LIMITATIONS}

The DD2 project has established a large population-based cohort of newly diagnosed patients with T2D, with demographic, anthropometric, lifestyle and clinical data. Furthermore, the project maintains a valuable biobank. Use of the unique individual-level CPR identifier makes it possible to avoid multiple registrations and to take advantage of existing data in Danish health and administrative registries in a cost-effective way. These registries also provide a source of long-term follow-up data. Since much of the data are recorded by health personnel during diagnosis and treatment, and not for research purposes, investigator bias is reduced. In addition, the validity of Danish registry data is high. As an example, reporting of cancer to the DCR is mandatory, and completeness is secured by cross-checking with other registries including DRCD and the Danish Pathology Registry. ${ }^{11}{ }^{21}$ In the DNPR, the positive predictive value of, for example, hospital discharge diagnoses included in the CCI is $94 \%-100 \% .^{22}$

A few limitations of the DD2 cohort must be noted. First, while DD2 and DDDA data are nearly complete for demographic variables and for many clinical variables such as smoking, physical activity, hip-waist ratio and resting heart rate, the proportion of missing data is large for some variables. For example, in the DDDA, BMI is missing for $26 \%$ of women. However, waist-hip ratio is available for $100 \%$ of the DD2 cohort and constitutes a better predictor of cardiovascular complications than BMI. ${ }^{23}$ Second, linkage to the DDDA currently is only possible for $73 \%$ of DD2 participants, partly explained by the data delay associated with the DDDA's role as a clinical quality improvement registry. Its mandate is to report on quality of care based on data collected during the previous year (ie, patients need to have prevalent T2D for 1-2 years before they are eligible for quality-of-care assessment). In contrast, the DD2 project focuses on incident T2D and is tasked with collecting data at time of diagnosis. ${ }^{7}$ Moreover, while reporting to the DDDA by GPs became mandatory in 2013, all GP reporting discontinued in September 2014 due to conflicting interpretation of legal issues concerning automated data transmission. Third, while it was planned for the DD2 cohort to recruit newly diagnosed T2D patients, approximately $85 \%$ already have initiated glucose-lowering treatment at enrolment. This hampers analyses of biomarkers among treatment-naive patients with T2D. However, the exact start date of glucose-lowering therapy can be ascertained from prescription registries. Fourth, since predominantly patients from outpatient specialist clinics were enrolled in early study years, that is, during 2010-2012, the cohort initially may have contained newly diagnosed patients with T2D with more advanced disease than average. However, the baseline data presented in this paper are similar to baseline data in a recent registry-based cohort study investigating patients with T2D from the Northern Region of Denmark at initiation of their first glucose-lowering therapy, ${ }^{24}$ thus reassuring that exposures of interest are represented for Danish patients withT2D in earlier phases of the disease. Finally, a large proportion of patients in the DD2 cohort were enrolled during the last few years, which limits current opportunities for conducting longterm follow-up studies.

In the future, the DD2 cohort will serve as a strong national and international resource for recruiting patients to nested case studies and clinical trials, postmarketing surveillance, large-scale genome studies, intervention 
studies, for example, in patients with rare diabetic subtypes, and follow-up studies of diabetes complications.

\section{COLLABORATION}

More information about the DD2 cohort can be found at the DD2 website www.dd2.nu. The DD2 project has a Steering Group with members from GP practices/ hospital research units in all regions of Denmark. The Steering Group strongly encourages national and international collaboration. Interested researchers can contact Director Dr Henning Beck-Nielsen at henning. beck-nielsen@syd.dk.

\author{
Author affiliations \\ ${ }^{1}$ Department of Clinical Epidemiology, Aarhus University Hospital, Aarhus, Denmark \\ ${ }^{2}$ Diabetes Research Centre, Department of Endocrinology, Odense University \\ Hospital, Odense, Denmark \\ ${ }^{3}$ Department of Pharmacology, University of Aarhus, Aarhus, Denmark \\ ${ }^{4}$ Department of Endocrinology, Bispebjerg, University Hospital Copenhagen, \\ Copenhagen, Denmark \\ ${ }^{5}$ Department of Endocrinology, Odense Universitetshospital, Odense, Denmark \\ ${ }^{6}$ Department of Biochemistry, Lillebaelt Hospital, Vejle, Denmark \\ ${ }^{7}$ Department of Internal Medicine and Endocrinology, Aarhus University Hospital, \\ Aarhus, Denmark \\ ${ }^{8}$ Cardiovascular and Metabolic Disease (CVMD) Translational Medicine Unit, Early \\ Clinical Development, IMED Biotech Unit, AstraZeneca, Gothenburg, Sweden \\ ${ }^{9}$ Department of Health Research \& Policy (Epidemiology), Stanford University, \\ Stanford, California, USA
}

Contributors HB-N, HTS, JSC, JR, AV, SF, IB, JSN and RWT designed the DD2 cohort and raised the funding. IB is in charge of storage and analyses of biological samples. SKN and KB performed all the statistical analyses. DHC and RWT wrote the first draft of this report. All authors critically revised the manuscript and approved the final version.

Funding The Danish Centre for Strategic Research in Type 2 Diabetes Project (DD2) is supported by the Danish Agency for Science (grant number 09-067009, 09-075724), the Danish Health and Medicines Authority, the Danish Diabetes Association, Sundhedsstyrelsen and an unrestricted donation from Novo Nordisk A/S. Project partners are listed on the website www.DD2.nu. Furthermore, this work was supported in part by the Program for Clinical Research Infrastructure (PROCRIN) established by the Lundbeck Foundation and the Novo Nordisk Foundation.

Competing interests The salary of DHC is paid by the International Diabetic Neuropathy Consortium (IDNC) research programme, which is supported by a Novo Nordisk Foundation Challenge Programme grant (grant number NNF140C0011633). AV started employment at AstraZeneca in March 2016. JSC has served on advisory boards and speaker panels for Novo Nordisk. The remaining authors report no personal conflicts of interest pertaining to this work. Moreover, the Department of Clinical Epidemiology, Aarhus University Hospital, participates in the IDNC research programme and is involved in the Program for Clinical Research Infrastructure (PROCRIN) established by the Lundbeck Foundation and the Novo Nordisk Foundation. The Department of Clinical Epidemiology, Aarhus University Hospital, also receives funding for other studies from companies in the form of research grants to (and administered by) Aarhus University. None of these studies have any relation to the present study.

Patient consent Detail has been removed from this case description/these case descriptions to ensure anonymity. The editors and reviewers have seen the detailed information available and are satisfied that the information backs up the case the authors are making.

Ethics approval The DD2 Project was approved by the Regional Committee on Health Research Ethics for Southern Denmark (Record number S-20100082) and the Danish Data Protection Agency (2008-58-0035).

Provenance and peer review Not commissioned; externally peer reviewed.

Data sharing statement See Collaboration section.
Open Access This is an Open Access article distributed in accordance with the Creative Commons Attribution Non Commercial (CC BY-NC 4.0) license, which permits others to distribute, remix, adapt, build upon this work non-commercially, and license their derivative works on different terms, provided the original work is properly cited and the use is non-commercial. See: http://creativecommons.org/ licenses/by-nc/4.0/

(C) Article author(s) (or their employer(s) unless otherwise stated in the text of the article) 2018. All rights reserved. No commercial use is permitted unless otherwise expressly granted.

\section{REFERENCES}

1. Rao Kondapally Seshasai S, Kaptoge S, Thompson A, et al. Diabetes mellitus, fasting glucose, and risk of cause-specific death. $N$ Engl J Med 2011;364:829-41.

2. Christensen DH, Rungby J, Thomsen RW. Nationwide trends in glucose-lowering drug use, Denmark, 1999-2014. Clin Epidemiol 2016;8:381-7.

3. Steffensen C, Thomsen RW, Vaag A, et al. The Danish Centre for Strategic Research in Type 2 Diabetes (DD2) Project: rationale and planned nationwide studies of genetic predictors, physical exercise, and individualized pharmacological treatment. Clin Epidemiol 2012;4:7-13.

4. Schmidt M, Pedersen L, Sørensen HT. The Danish civil registration system as a tool in epidemiology. Eur J Epidemiol 2014;29:541-9.

5. Nielsen JS, Thomsen RW, Steffensen C, et al. The Danish Centre for Strategic Research in Type 2 Diabetes (DD2) study: implementation of a nationwide patient enrollment system. Clin Epidemiol 2012;4:27-36.

6. Christensen H, Nielsen JS, Sørensen KM, et al. New national Biobank of The Danish Center for Strategic Research on Type 2 Diabetes (DD2). Clin Epidemiol 2012;4:37-42.

7. Thomsen RW, Friborg S, Nielsen JS, et al. The Danish centre for strategic research in type 2 diabetes (DD2): organization of diabetes care in Denmark and supplementary data sources for data collection among DD2 study participants. Clin Epidemiol 2012;4:15-19.

8. Schmidt M, Schmidt SA, Sandegaard JL, et al. The Danish National Patient Registry: a review of content, data quality, and research potential. Clin Epidemiol 2015;7:449-90.

9. Charlson ME, Pompei P, Ales KL, et al. A new method of classifying prognostic comorbidity in longitudinal studies: development and validation. J Chronic Dis 1987;40:373-83.

10. Johannesdottir SA, Horváth-Puhó E, Ehrenstein V, et al. Existing data sources for clinical epidemiology: The Danish national database of reimbursed prescriptions. Clin Epidemiol 2012;4:303-13.

11. Gjerstorff ML. The Danish cancer registry. Scand J Public Health 2011;39:42-5.

12. Helweg-Larsen K. The Danish register of causes of death. Scand J Public Health 2011;39:26-9.

13. Andersen JS, Olivarius NF, Krasnik A. The Danish national health service register. Scand J Public Health 2011;39:34-7.

14. Lab News 6/2015. Change in Proinsulin C-peptide analysis. Department of Biochemistry, Lillebaelt Hospital, Vejle, Denmark. http://www.sygehuslillebaelt.dk/wm473198 (accessed Aug 2016).

15. Mor A, Svensson E, Rungby J, et al. Modifiable clinical and lifestyle factors are associated with elevated alanine aminotransferase levels in newly diagnosed type 2 diabetes patients: results from the nationwide DD2 study. Diabetes Metab Res Rev 2014;30:707-15.

16. Svensson E, Mor A, Rungby J, et al. Lifestyle and clinical factors associated with elevated C-reactive protein among newly diagnosed Type 2 diabetes mellitus patients: a cross-sectional study from the nationwide DD2 cohort. BMC Endocr Disord 2014;14:74.

17. Mor A, Berencsi K, Svensson E, et al. Prescribing practices and clinical predictors of glucose-lowering therapy within the first year in people with newly diagnosed Type 2 diabetes. Diabet Med 2015;32:1546-54.

18. Svensson E, Berencsi K, Sander S, et al. Association of parental history of type 2 diabetes with age, lifestyle, anthropometric factors, and clinical severity at type 2 diabetes diagnosis: results from the DD2 study. Diabetes Metab Res Rev 2016;32:308-15.

19. Steffensen $\mathrm{C}$, Thomsen HH, Dekkers OM, et al. Low positive predictive value of midnight salivary cortisol measurement to detect hypercortisolism in type 2 diabetes. Clin Endocrinol 2016;85:202-6.

20. Ried-Larsen M, Thomsen RW, Berencsi K, et al. Implementation of interval walking training in patients with type 2 diabetes in Denmark: rationale, design, and baseline characteristics. Clin Epidemiol 2016;8:201-9. 
21. Storm HH, Michelsen EV, Clemmensen IH, et al. The Danish Cancer Registry--history, content, quality and use. Dan Med Bull 1997;44:535-9.

22. Thygesen SK, Christiansen CF, Christensen S, et al. The predictive value of ICD-10 diagnostic coding used to assess Charlson comorbidity index conditions in the population-based Danish National Registry of Patients. BMC Med Res Methodol 2011;11:83.
23. Czernichow S, Kengne AP, Huxley RR, et al. Comparison of waistto-hip ratio and other obesity indices as predictors of cardiovascular disease risk in people with type-2 diabetes: a prospective cohort study from ADVANCE. Eur J Cardiovasc Prev Rehabil 2011;18:312-9.

24. Thomsen RW, Baggesen LM, Svensson E, et al. Early glycaemic control among patients with type 2 diabetes and initial glucoselowering treatment: a 13-year population-based cohort study. Diabetes Obes Metab 2015;17:771-80. 